\title{
Using Fullwave Simulations to Understand the Turbulent Wavenumber Spectrum Measured by Doppler Reflectometry
}

\author{
C. Lechte ${ }^{1}$, G. D. Conway ${ }^{2}$, T. Görler ${ }^{2}$, T. Happel ${ }^{2}$, C. Tröster-Schmid ${ }^{2}$, the ASDEX Upgrade \\ Team
}

${ }^{1}$ IGVP, University of Stuttgart, Germany, carsten.lechte@igvp.uni-stuttgart.de ${ }^{2}$ Max-Planck-Institut für Plasmaphysik, Boltzmannstr. 2, 85748 Garching, Germany

\begin{abstract}
Doppler reflectometry is an important microwave diagnostic for turbulent fusion plasma. A low power probing beam between 40 and $100 \mathrm{GHz}$ in $\mathrm{O}$ or $\mathrm{X}$ mode polarisation is scattered at certain density fluctuation wavenumbers, depending on frequency and angle between beam and density gradient. This way, a radially localised wavenumber resolved density fluctuation spectrum can be measured. However, the correspondence between fluctuation power and scattered microwave power is strongly non-linear and is investigated using fullwave simulations, with density fluctuations supplied by a plasma turbulence code. Earlier results for $\mathrm{X}$ mode polarisation have shown saturation of the spectral power to occur at high fluctuation amplitudes, an enhancement at intermediate amplitudes, and linear behaviour at low amplitudes. Together, these effects strongly modify the shape of the wavenumber spectrum [1].
\end{abstract}

Experimental data in $\mathrm{X}$ and $\mathrm{O}$ mode polarisation and accompanying plasma fluctuation data from the GENE code have been used to simulate the Doppler reflectometer response with the fullwave code IPFFD3D [2].

The gyrokinetic plasma turbulence code GENE is a physically comprehensive, hyperscalable Vlasov code that is used with the same ASDEX Upgrade equilibrium and plasma profiles as the experiment and the fullwave simulations. A flux tube surrounding the radial position of the microwave cutoff was simulated. The input parameters were adjusted slightly until the resultant turbulent fluxes matched the ones observed in experiment. The output density fluctuation fields are used as inputs in the fullwave simulations.

IPF-FD3D is a finite difference time domain code for cold plasmas. Simulations were done in $2 \mathrm{D}$ for both polarisations in the perpendicular plane for 512 different turbulence time slices. The turbulence is considered frozen in time while the wave is propagating. The antenna angles and frequencies were adjusted to probe the same $\rho_{\mathrm{pol}}$ as the experiment. The setup is shown in Fig. 1.

Comparison with experimental data showed a good agreement [2], with the O mode spectrum corresponding more closely to the GENE density spectrum. Furthermore, the fullwave simulation data in Fig. 2 shows the big difference between $\mathrm{O}$ and $\mathrm{X}$ mode polarisation, which were both probing the same fluctuations. The spectrum derived from $\mathrm{O}$ mode scattering looks similar to the real spectrum of the density fluctuations, albeit with power law exponent -8.7 instead of -6.7 . The $\mathrm{X}$ mode spectrum is much shallower at the nominal fluctuation amplitude taken directly from GENE. In addition, the kink or knee of the spectrum is moved to larger wavenumbers compared to $\mathrm{O}$ mode and GENE. The knee should be the same, as it is determined by the instability that is driving the turbulence.

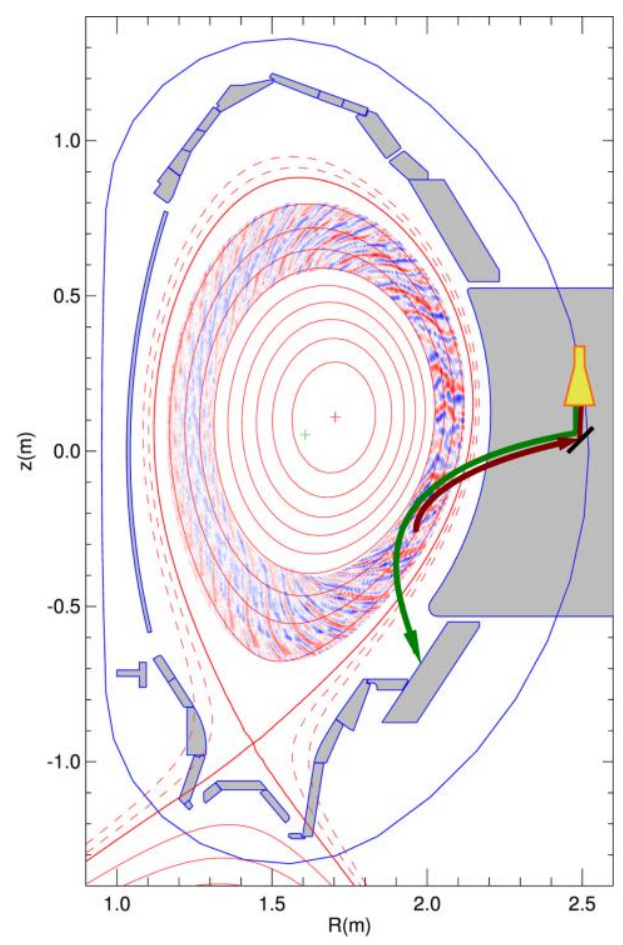

Fig. 1. Poloidal geometry of ASDEX Upgrade with Doppler reflectometer beams and superimposed density fluctuation field from GENE

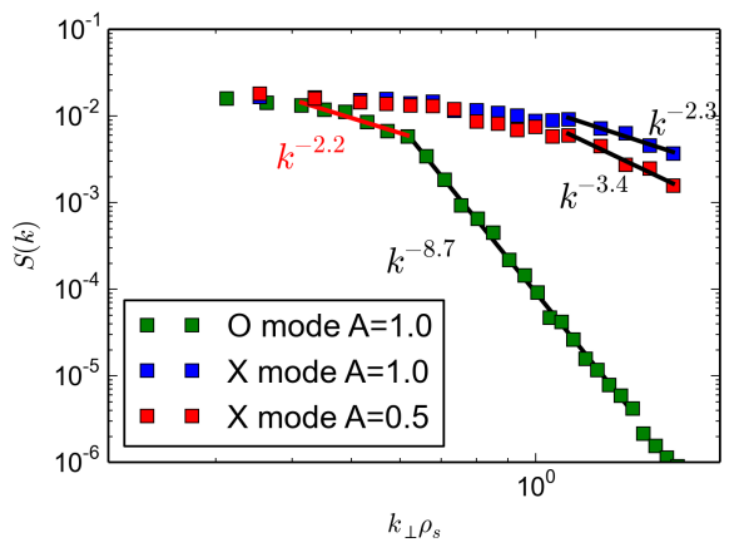

Fig. 2. Wavenumber spectra from fullwave Doppler reflectometry simulations for both $\mathrm{X}$ and $\mathrm{O}$ mode polarisation with fitted spectral indices. 


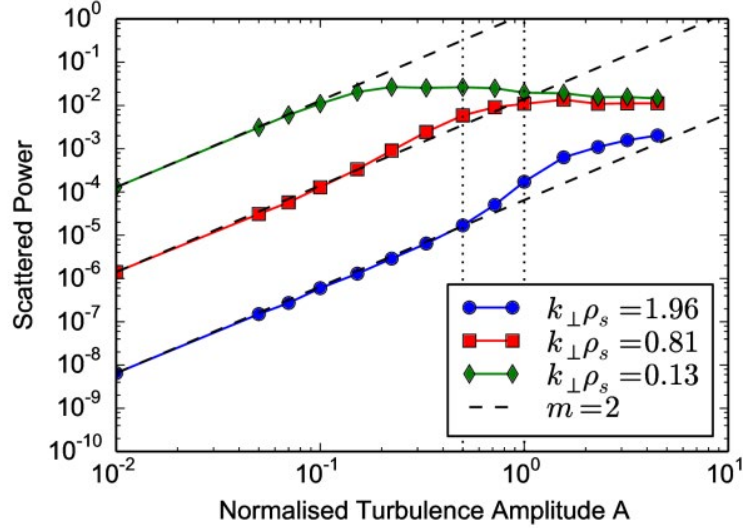

Fig. 3. The scattered power plotted over the density fluctuation amplitude for 3 exemplary wavenumbers for $\mathrm{X}$ mode polarisation

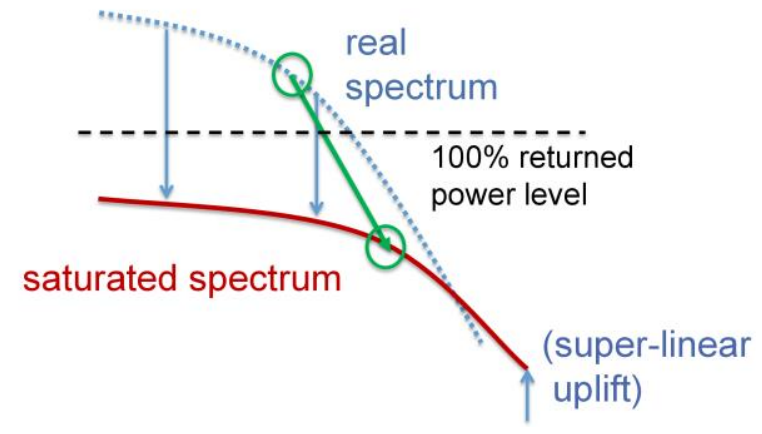

Fig. 4. Schematic wavenumber spectra illustrating the observed deformation of the spectrum caused by the sensitivity shifts shown in Fig. 3

A factor of 0.5 was necessary to bring the simulated Doppler spectrum in line with the experimental one. This is justified because of some uncertainties in the temperature profiles.

Scaling down the fluctuation amplitude reveals steeper spectra, which also do not have the saturation effect at low wavenumbers. This means that for $\mathrm{X}$ mode, the density fluctuations are so large that the spectrum is non-linearly saturated, while for $\mathrm{O}$ mode, it is still in the linear regime. This was simulated in more detail with simple isotropic turbulence, and is shown in Fig. 3.

The scan of fluctuation amplitudes (see Fig. 3) shows that a non-linear saturation sets in at a scattered power of 0.01 a.u. Due to the high level of low- $\mathrm{k}_{\perp}$ fluctuations, this part of the spectrum is saturated, while the high- $\mathrm{k}_{\perp}$ fluctuations follow a linear relation, but with a non-linear enhancement. This had already been reported in [3]. The turbulence amplitude $\mathrm{A}$ is normalised to the GENE results.

The overall effect on the spectrum for $\mathrm{X}$ mode Doppler reflectometry is illustrated in Fig. 4. The shape of the density fluctuation spectrum (labeled 'real spectrum') is strongly decreased at the low- $\mathrm{k}_{\perp}$ high-amplitude regime because of non-linear saturation. At the high- $\mathrm{k}_{\perp}$-low-amplitude end of the spectrum, we see the non-linear enhancement. The overall effect is a much shallower spectrum with the knee position apparently shifted to higher $\mathrm{k}_{\perp}$ (indicated by the green arrow).

In $\mathrm{O}$ mode polarisation, the non-linear saturation effect is much less pronounced, resulting in a much steeper spectrum. This is attributed to the lower frequencies that are necessary to probe the same wavenumbers in $\mathrm{O}$ mode.

In summary, the discrepancy between the GENE density fluctuation spectra and the Doppler reflectometry wavenumber spectra can be explained by the nonlinear saturation and enhancement properties of the diagnostic itself. This also helps validate the turbulence simulations.

\section{Acknowledgements}

The simulations were performed on the HPC-FF computer cluster in Jülich, Germany; on the BOB Linux cluster at RZG; on hornet and hazelhen at HLRS Stuttgart; on bwUniCluster funded by the State and the universities of Baden-Württemberg, Germany, within the framework programme bwHPC. This work was also partly performed within the framework of the Helmholtz Virtual Institute on Plasma Dynamical Processes and Turbulence Studies using Advanced Microwave Diagnostics.

\section{References}

[1] C. Lechte et al. "X mode Doppler Reflectometry kspectral measurements in ASDEX Upgrade: Experiments and simulations" Plasma Phys. Contr. Fus. 59 (7) 2017 doi:10.1088/1361-6587/aa6fe7

[2] T. Happel et al. "Comparison of detailed experimental wavenumber spectra with gyrokinetic simulation aided by two-dimensional full-wave simulations" Plasma Phys. Contr. Fus. 59 (5) 2017 doi:10.1088/1361-6587/ aa645b

[3] J. R. Pinzón et al. „Enhanced Doppler reflectometry power response: physical optics and 2D full wave modelling" Plasma Phys. Contr. Fus. 59 2017, doi:10.1088/1361$6587 / \mathrm{aa} 543 \mathrm{c}$ 